\title{
Optimización del proceso RSW para acero DP 1000
}

\author{
César David Marconi ${ }^{1}$, María José Castillo ${ }^{1,2}$, Carlos Fernando Consigli ${ }^{1}$, \\ Hernán Gabriel Svoboda $1,3,4$
}

\author{
${ }^{1}$ DEYTEMA-Centro de Desarrollo y Tecnología de Materiales, Facultad Regional San Nicolás, Universidad Tecnológica \\ Nacional, San Nicolás, Buenos Aires, Argentina. e-mail: cmarconi@frsn.utn.edu.ar \\ ${ }^{2}$ Escuela de Tecnología, UNNOBA, Junín, Buenos Aires, Argentina. \\ ${ }^{3}$ GTSyCM3, INTECIN, Facultad de Ingeniería, Universidad de Buenos Aires-CONICET, Buenos Aires, Buenos Aires, \\ Argentina. \\ ${ }^{4}$ Consejo Nacional de Investigaciones Científicas y Técnicas, Buenos Aires, Buenos Aires, Argentina. \\ e-mail: mariajose.castillo@nexo.unnoba.edu.ar; hsvobod@gmail.com
}

\section{RESUMEN}

En el último tiempo se han introducido nuevos materiales en la construcción de automóviles a fin de disminuir su peso y consecuentemente el consumo de combustible y el nivel de emisiones. Entre ellos se encuentran los Aceros Dual Phase (DP), constituidos por una matriz ferrítica con islas de martensita. La soldadura por puntos de resistencia (RSW) es el proceso de soldadura más utilizado en la industria automotriz. Sin embargo, la soldadura de estos materiales presenta mayores desafíos, ya que los parámetros empleados para la soldadura RSW de aceros convencionales no son aplicables a estos aceros avanzados. Asimismo, los ciclos térmicos de la soldadura degradan sus propiedades encontrándose el mínimo de dureza en la zona sub y/o inter crítica. A su vez, debido a las altas velocidades de enfriamiento impuestas por la fuerte refrigeración de los electrodos, en la zona de la lenteja se pueden generar estructuras de elevada dureza. En este sentido, la optimización de los procedimientos de soldadura presenta un interés especial. Por otro lado, la productividad es un aspecto de gran relevancia en estas aplicaciones por lo que la reducción de los tiempos de soldadura es un requerimiento. El objetivo de este trabajo es evaluar el efecto de los parámetros de soldadura RSW en aceros DP1000 de forma de optimizar el mismo. A este fin, se soldaron mediante RSW probetas de acero DP1000 de 1,2 mm de espesor, variando la corriente y el tiempo de soldadura. Sobre las uniones soldadas se realizó la caracterización macro y microestructural mediante microscopía óptica y electrónica de barrido, perfiles de microdureza Vickers y ensayos de arrancamiento tipo peel-test y shear-test. Las superficies de fractura se analizaron mediante microscopía óptica. Se obtuvo un conjunto de parámetros que optimiza el procedimiento de soldadura de aceros DP1000 mediante RSW. Se encontró que a partir de los 10 ciclos de soldadura y con niveles de corriente de $7 \mathrm{KA}$ los puntos cumplen con los requerimientos dimensionales y de propiedades mecánicas adoptados por la industria automotriz.

Palabras clave: Soldadura de Punto por Resistencia, Aceros Dual Phase , Microestructura, Propiedades Mecánicas, Optimización.

\section{ABSTRACT}

In recent times new materials have been introduced in automobiles construction in order to reduce their weight and consequently fuel consumption and emissions level. Among them are Dual Phase (DP) steels, constituted by a ferritic matrix with martensite islands. Resistance Spot Welding (RSW) is the most commonly used welding process in the automotive industry. However, the welding of these materials presents greater challenges, since the parameters used for RSW welding of conventional steels are not applicable on these advanced steels. Also, the welding thermal cycle degraded its properties, with the minimum hardness being found in the sub and/or inter critical zone. In turn, due to the high cooling rates imposed by the strong cooling of the electrodes, structures of high hardness can be generated in the nugget region. In this sense, the optimization of welding procedures is of particular interest. In other way, productivity is an great relevance aspect in these applications, thus reducing welding times is a requirement. The objective of this work is to evaluate the effect of RSW welding parameters on DP1000 steels in order to optimize them. To this end, $1.2 \mathrm{~mm}$ thick DP1000 steel probes were welded by RSW, varying the current and the welding time. On the welded joints, 
macro and microstructural characterization was performed by optical and scanning electron microscopy, Vickers microhardness profiles and peel-test and shear-test stripping tests. The fracture surfaces were analyzed by light microscopy. A set of parameters that optimizes the welding process of DP1000 steels by RSW was obtained. It was found that from 10 welding cycles and with current levels of 7 KA the points achieves the dimensional requirements and mechanical properties adopted by the automotive industry.

Keywords: Resistance Spot Welding, Dual Phase Steel, Microstructure, Mechanicals Properties, Optimization.

\section{INTRODUCCIÓN}

En los últimos años, la industria automotriz se ha orientado al desarrollo de vehículos con mayores niveles de seguridad, menores consumos de combustible y métodos de producción rentables. Además, existe la necesidad real de reducir los niveles de emisión de $\mathrm{CO}_{2}$. Una de las alternativas para lograr esto es utilizar materiales de mayor resistencia mecánica, a fin de disminuir los espesores empleados en partes estructurales. En este contexto, surgen los Aceros Avanzados de Alta Resistencia (AHSS) [1,2]. Dentro de esta familia de aceros, los aceros de fase dual (Dual Phase - DP) ofrecen una buena combinación de resistencia, conformabilidad y capacidad de absorción de energía. Esto, unido a la rentabilidad del conformado en frío, se traduce en soluciones altamente atractivas para piezas estructurales de automóviles. Los aceros DP consisten de un 5-50\% de islas de martensita en una matriz de ferrita, además de un porcentaje bajo de bainita y austenita retenida. La segunda fase martensítica aumenta con el aumento de la resistencia deseada del acero (500 a $1200 \mathrm{MPa}$ ). La fase ferrítica blanda es generalmente continua, proveyendo una excelente ductilidad. El contenido de carbono y de aleantes, los tamaños de grano y la fracción de martensita, entre otros aspectos, determinarán las propiedades finales del material. El desarrollo de estos aceros cuenta ya con varias décadas, sin embargo recién en los últimos años se han incorporado fuertemente para la fabricación de partes estructurales en la industria automotriz, en buena medida por el desarrollo de procesos para su obtención y soldadura. Sin embargo, junto con las ventajas que proveen estos nuevos aceros surge la necesidad de profundizar en aspectos como su soldabilidad [3,4].

En los aceros DP las altas resistencias se obtienen a partir de la modificación de la microestructura, la cual se verá afectada por el ciclo térmico de la soldadura. Para la soldadura de estos materiales se debe tener especial control sobre los parámetros del proceso que afecten el aporte térmico y la evolución microestructural, ya que ésta definirá las propiedades mecánicas resultantes. Así la comprensión de los aspectos metalúrgicos que controlan la mencionada evolución microestructural es de fundamental importancia a fin de poder predecir el comportamiento mecánico de las uniones soldadas obtenidas. Para los aceros DP, en general, como consecuencia de la soldadura se produce en la zona afectada por el calor (ZAC) cercana al metal base, el revenido de la fase martensítica, por lo que la dureza en dichas zonas disminuye. La resistencia de la unión soldada presenta, en consecuencia, una disminución en comparación con la del metal base, debido a la formación de las regiones blandas en la ZAC. Adicionalmente, se ha mostrado que cuanto mayor es la resistencia del acero DP, mayor es la disminución de la dureza en la ZAC [5,6].

La soldadura por puntos de resistencia (RSW) es el principal proceso de soldadura de chapa metálica en la fabricación de automóviles. La infraestructura integrada hace del RSW un proceso económicamente deseable. Los modos de fractura en soldadura RSW de aceros son extracción de botón, extracción parcial y fractura interfacial, donde se prefiere la extracción del botón ya que se absorbe más energía durante este tipo de falla. Se ha encontrado que el tipo de fractura depende principalmente del tamaño de la zona de fusión o lenteja y la falla tiende a ocurrir en modo de extracción a medida que aumenta el tamaño de la misma. El tamaño de la lenteja de soldadura es de hecho el principal parámetro variable con el aumento de la intensidad de la corriente de soldadura durante la determinación del intervalo de soldadura para una configuración de chapa dada. Algunos autores se centraron en la transición del modo de fractura con el aumento del diámetro de la soldadura, proponiendo relaciones analíticas simples que expresan la transición de la fractura interfacial a la extracción de botones. También se ha informado acerca de la formación de huecos de contracción en la zona de fusión de aceros DP600 soldado por puntos, formados debido a una composición química más rica de los aceros DP en comparación con los aceros con bajo contenido de carbono. Diversos trabajos concluyeron que la tenacidad a la fractura de la soldadura RSW depende del diámetro de la lenteja, el espesor de la chapa, la carga de rotura por tracción, el tiempo de soldadura y la corriente [7-9].

Se sabe que los parámetros geométricos de los puntos de soldadura influyen directamente en su respuesta mecánica. Entre estos parámetros, el diámetro de la lenteja de soldadura y el espesor de la chapa correspondiente están entre los más importantes y afectan fuertemente el comportamiento mecánico de las soldaduras por puntos. Numerosos autores los utilizan directamente para expresar la resistencia de la soldadura 
por puntos. De acuerdo con las normas industriales, el tamaño mínimo de las soldaduras por puntos para garantizar la fractura extraíble sigue la relación $\mathrm{D}=\mathrm{K} \sqrt{\mathrm{t}}$, donde $\mathrm{D}$ es el diámetro de la lenteja de soldadura en $\mathrm{mm}, \mathrm{K}$ es una constante dependiente del proceso que va de 3 a 6 , y t es el espesor mínimo de la chapa en mm $[10,11]$.

El objetivo de este trabajo es evaluar el efecto de los parámetros de soldadura RSW en aceros DP1000 de forma de optimizar el mismo. Asimismo, se busca profundizar en la comprensión de la relación entre parámetros de soldadura, microestructura/defectos y propiedades para este tipo de materiales.

\section{MATERIALES Y MÉTODOS}

\subsection{Caracterización del metal base}

Como metal base se utilizó un acero DP de $1000 \mathrm{MPa}$ de resistencia a la tracción de 1,2 mm de espesor, sobre el cual se determinó la composición química mediante espectrometría de emisión óptica (OES) y se realizó la caracterización microestructural mediante microscopia óptica (LM) y electrónica de barrido (SEM). Asimismo, se determinaron las propiedades mecánicas mediante mediciones de microdureza Vickers (HV1Kg) y ensayo de tracción uniaxial, según la Norma ASTM E8-04. En la Tabla 1 se muestra la composición química del material empleado expresada en porcentaje en peso (wt\%).

Tabla 1: Composición química del metal base (wt\%)

\begin{tabular}{l|l|l|l|l|l|l|l|l|l|l|l|l|l}
\hline MUESTRA & $\mathbf{C}$ & Mn & Si & P & S & Nb & Ti & V & $\mathbf{B}^{*}$ & Al & Cr & Mo & Cu \\
\hline DP1000 & 0,14 & 1,49 & 0,48 & 0,009 & $<0,001$ & 0,016 & 0,003 & 0,006 & 3 & 0,039 & 0,03 & 0,01 & 0,01 \\
\hline
\end{tabular}

*expresado en ppm

Puede verse que corresponde a un acero de bajo contenido de $\mathrm{C}$ aleado al $\mathrm{Mn}$ y $\mathrm{Si}$, con la presencia de microaleantes como el $\mathrm{Nb}$. Cuanto más alto el contenido de $\mathrm{C}$ y $\mathrm{Mn}$, en comparación con chapas de acero convencionales, se mejora la capacidad de obtener el endurecimiento necesario, mediante la prevención de perlita o bainita que se forman durante el procesamiento. El Si permite el enriquecimiento de la partición de carbono en la austenita [3].

En la Tabla 2 se muestran los resultados de las propiedades mecánicas del material base empleado.

Tabla 2: Propiedades mecánicas del metal base HV1: Microdureza Vickers (1 kg). R: resistencia a la tracción. Rf: tensión de fluencia. A: alargamiento.

\begin{tabular}{l|l|l|l}
\hline HV1 & R (MPa) & Rf (Mpa) & A (\%) \\
\hline 309 & 1032 & 753 & 10 \\
\hline
\end{tabular}

Se observa que corresponde a un material de alta resistencia, superando los $1000 \mathrm{MPa}$ de resistencia a la tracción con una relación de $\mathrm{R} / \mathrm{Rf}$ de 1,37, con un buen alargamiento a rotura.

En la Figura 1 se muestra la microestructura del acero base obtenida mediante LM y SEM. Se aprecian las islas de martensita en una matriz de ferrita y tamaño de grano fino uniforme menor a $10 \mu \mathrm{m}$ de diámetro en todo el espesor de la chapa medido según la Norma ASTM E 112-96. El contenido de cada fase determinado según la Norma ASTM E 562-02 corresponde con un $57 \%$ de ferrita y un $43 \%$ de martensita.
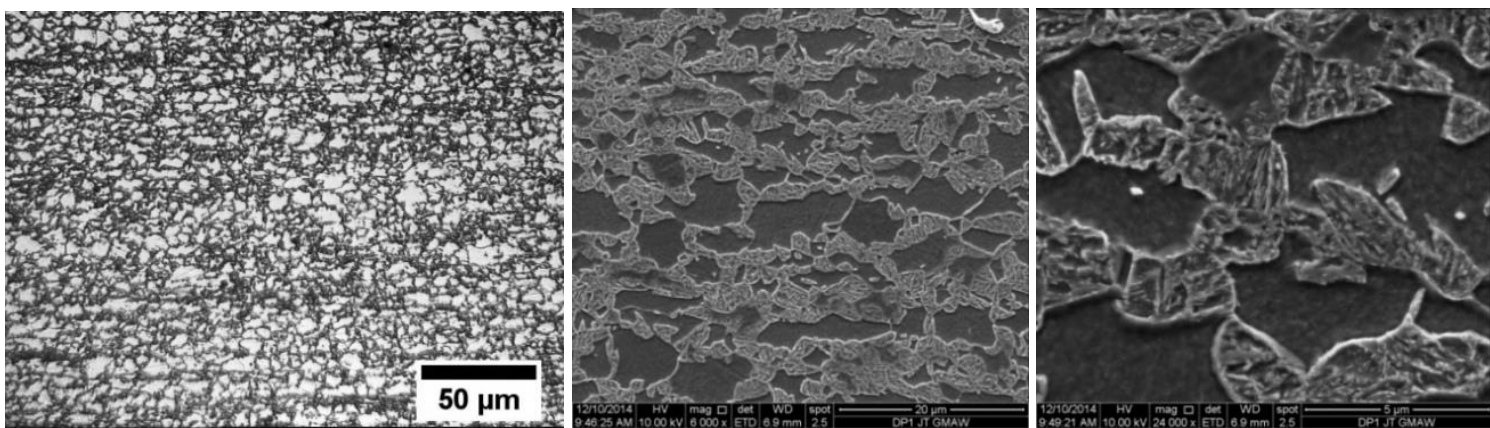

Figura 1: Microestructura del acero base, (a) microscopia óptica; (b y c) electrónica de barrido 


\subsection{Ejecución de uniones soldadas}

A fin de alcanzar los objetivos propuestos se soldaron probetas de acero DP 1000 mediante el proceso RSW bajo distintas configuraciones operativas. Para la soldadura de las probetas se utilizó una máquina de pedestal de $30 \mathrm{KVa}$ y se realizaron los puntos de junta soldada en chapas solapadas de 30x30 mm, según la Especificación ISO 18278-2 [12]. En la Figura 2 se muestra el equipo empleado.

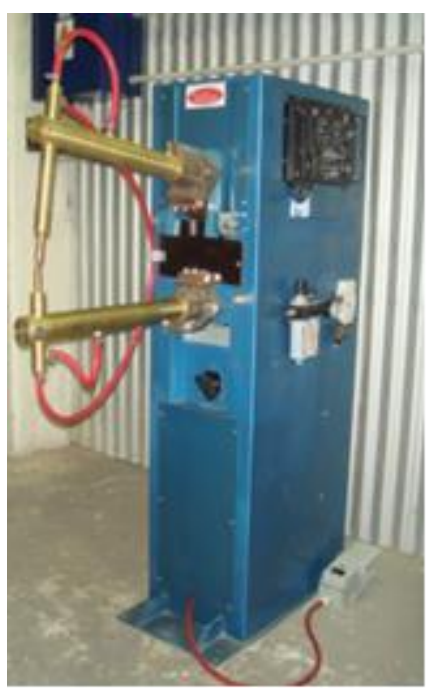

Figura 2: Equipamiento empleado para la soldadura de las chapas de acero DP 1000

El caudal de agua refrigeración fue de $4 \mathrm{~L} / \mathrm{min}$. Los electrodos utilizados fueron de una aleación $\mathrm{Cu}$ Cr con una cara activa de diámetro $6 \mathrm{~mm}$. La fuerza de apriete entre electrodos fue de $2 \mathrm{kN}$, para todos los casos.

Antes de definir el esquema de parámetros para realizar el estudio sistemático se realizaron varias pruebas hasta encontrar una ventana de soldadura con buen aspecto superficial y sin defectos macroscópicos. Se mantuvieron constantes los tiempos de presoldadura (50 ciclos) y postsoldadura (20 ciclos) y los parámetros que se variaron fueron la corriente (5, 6 y $7 \mathrm{KA})$, y los ciclos de soldadura $(4,6,10,30$ y 50 ciclos). La identificación de cada probeta con sus respectivos parámetros de soldadura utilizados se muestra en la Tabla 3.

Tabla 3: Parámetros de soldadura e identificación de las probetas

\begin{tabular}{l|l|l}
\hline PROBETAS & $\begin{array}{l}\text { SOLDADURA } \\
\text { (CICLOS) }\end{array}$ & $\begin{array}{l}\text { CORRIENTE } \\
\text { (KA) }\end{array}$ \\
\hline $\mathbf{4}-\mathbf{5}$ & 4 & 4,3 \\
\hline $\mathbf{6}-\mathbf{5}$ & 6 & 4,5 \\
\hline $\mathbf{1 0}-\mathbf{5}$ & 10 & 4,8 \\
\hline $\mathbf{3 0}-\mathbf{5}$ & 30 & 5,5 \\
\hline $\mathbf{5 0}-\mathbf{5}$ & 50 & 5,9 \\
\hline $\mathbf{4}-\mathbf{6}$ & 4 & 6,2 \\
\hline $\mathbf{6}-\mathbf{6}$ & 6 & 6,4 \\
\hline $\mathbf{1 0}-\mathbf{6}$ & 10 & 6,7 \\
\hline $\mathbf{3 0}-\mathbf{6}$ & 30 & 7,2 \\
\hline $\mathbf{5 0}-\mathbf{6}$ & 50 & 7,6 \\
\hline $\mathbf{4}-\mathbf{7}$ & 4 & 7,2 \\
\hline $\mathbf{6}-\mathbf{7}$ & 6 & 7,5 \\
\hline $\mathbf{1 0}-\mathbf{7}$ & 10 & 7,8 \\
\hline $\mathbf{3 0}-\mathbf{7}$ & 30 & 8,4 \\
\hline $\mathbf{5 0}-\mathbf{7}$ & 50 & 9 \\
\hline
\end{tabular}

En cada condición se soldaron probetas para análisis microestructural, ensayos de arrancamiento (peel) y corte (shear). 


\subsection{Análisis Dimensional y Microestructural}

Sobre las probetas soldadas se realizó una inspección visual y se evaluó el aspecto superficial de los puntos y el nivel de expulsiones.

Se realizaron cortes transversales a los puntos los que se prepararon para observación metalográfica. Todas las muestras se desbastaron con papeles abrasivos de carburo de silicio hasta grado 600, el pulido final se realizó utilizando pasta diamantada de 6 y $1 \mu \mathrm{m}$. La macroestructura de soldadura se reveló con Nital $2 \%$ y se obtuvieron imágenes con lupa estereoscópica Olympus SX61. Sobre los puntos se realizó un análisis dimensional empleando el software de análisis de imágenes ImageJ, relevando las dimensiones mostradas en el esquema de la Figura 3.

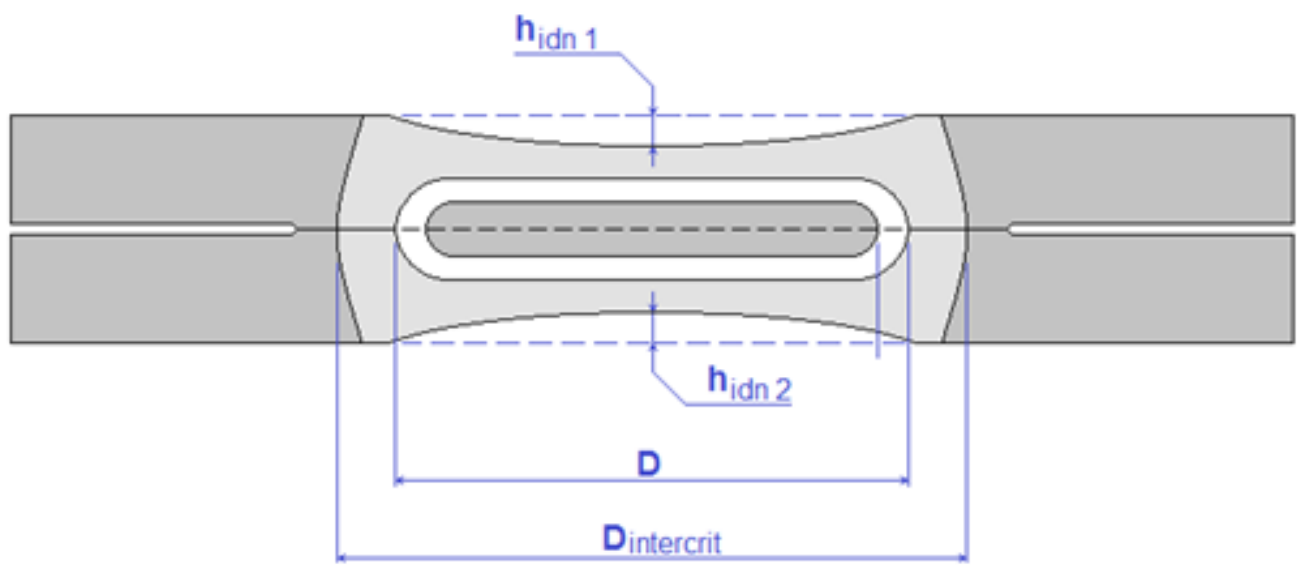

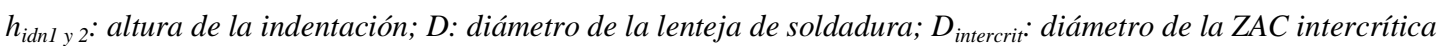

Figura 3: Esquema de las mediciones tomadas en las soldaduras

Mediante LM y SEM se analizó la evolución microestructural del punto de soldadura. Se partió desde el metal base (MB), luego se dividió la ZAC en 4 zonas: Zona de Metal Base Revenido (ZMBR), Zona SubCrítica (ZSC), Zona Inter-Crítica (ZIC) y Zona Recristalizada Fina (ZRF) y Gruesa (ZRG), respectivamente. Por último se caracterizó el metal de soldadura (MS) o lenteja de soldadura.

\subsection{Microdureza}

Se realizaron perfiles de microdureza Vickers (HV300g) con una distancia entre improntas de $25 \mu \mathrm{m}$ con un microdurómetro LECO LM 300 AT. Las improntas fueron realizadas a través de la diagonal de los puntos y desde el metal base. El esquema de la Figura 4 indica el procedimiento para la determinación.

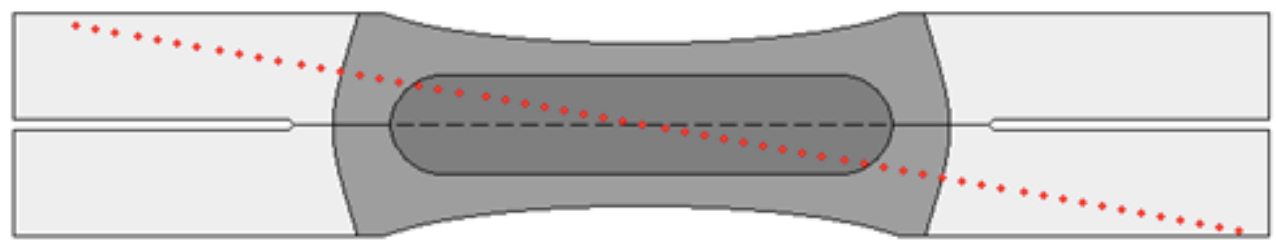

Figura 4: Esquema de los perfiles de microdureza en la sección transversal de los puntos de soldadura

\subsection{Ensayos de Arrancamiento y Corte}

Las probetas soldadas para los ensayos de arrancamiento y corte (peel y shear test) se realizaron según las Normas ISO 14270 y 14273 [13,14], de acuerdo al esquema de la Figura 5. Las mismas fueron ensayadas con una máquina de tracción Tinius Olsen H50KT. 


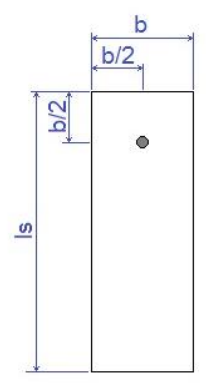

a

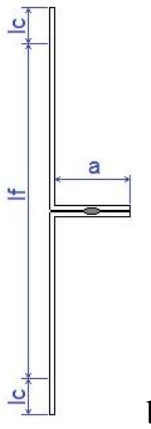

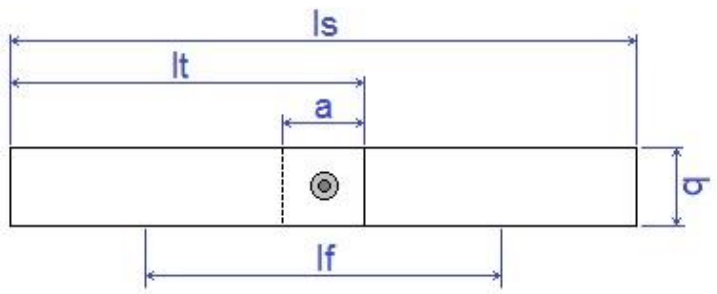

Figura 5: Esquema de las probetas para los ensayos de: a- arrancamiento (peel) y b- corte (shear)

Sobre cada probeta ensayada se tomaron fotomicrografías y se analizó el modo de fractura para las distintas condiciones de soldadura. Se caracterizaron las fracturas de acuerdo a tres tipos: desprendimiento interfacial, arranque parcial y arranque total.

\section{RESULTADOS}

\subsection{Inspección visual y análisis dimensional}

En la Figura 6 se muestran las fotomacrografías de los puntos de soldadura obtenidos, observándose el aspecto superficial de cada uno de ellos.

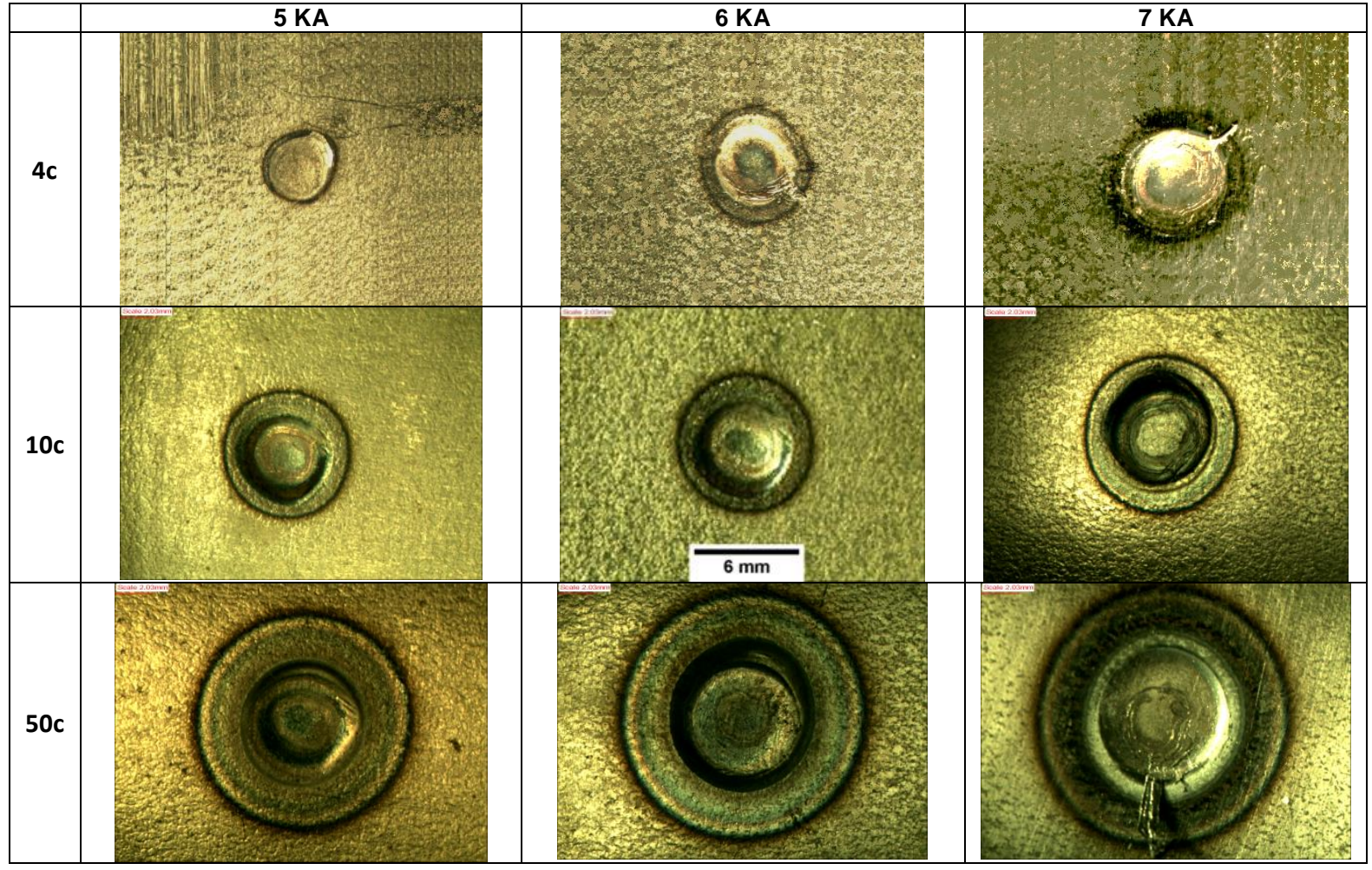

Figura 6: Aspecto superficial de los puntos de soldadura

En general se observa que los puntos no presentaron imperfecciones visibles, ni excesivas expulsiones de material, presentando una muy buena apariencia superficial a partir de los 6 ciclos. También se puede apreciar que el tamaño de la ZAC aumenta con la corriente y sobre todo con el tiempo de soldadura. En la Tabla 4 se muestran los resultados del análisis dimensional para todas las configuraciones analizadas. 
Tabla 4: Resultados del análisis dimensional

\begin{tabular}{l|l|l|l}
\hline MUESTRAS & $\begin{array}{l}\text { DIÁMETRO DE LA } \\
\text { LENTEJA (MM) }\end{array}$ & $\begin{array}{l}\text { ANCHO ZAC } \\
\text { INTERCRÍTICO } \\
\text { (MM) }\end{array}$ & $\begin{array}{l}\text { INDENTACIÓN } \\
\text { PROMEDIO } \\
\text { (MM) }\end{array}$ \\
\hline $\mathbf{4}-\mathbf{5}$ & 2,4 & 4,5 & 0,04 \\
\hline $\mathbf{6}-\mathbf{5}$ & 3,2 & 5,5 & 0,07 \\
\hline $\mathbf{1 0}-\mathbf{5}$ & 4,5 & 6,8 & 0,10 \\
\hline $\mathbf{3 0}-\mathbf{5}$ & 4,8 & 9,0 & 0,16 \\
\hline $\mathbf{5 0}-\mathbf{5}$ & 5,4 & 10,5 & 0,18 \\
\hline $\mathbf{4}-\mathbf{6}$ & 3 & 5,0 & 0,08 \\
\hline $\mathbf{6}-\mathbf{6}$ & 4,2 & 6,2 & 0,10 \\
\hline $\mathbf{1 0}-\mathbf{6}$ & 6,1 & 7,4 & 0,13 \\
\hline $\mathbf{3 0}-\mathbf{6}$ & 6,1 & 9,9 & 0,16 \\
\hline $\mathbf{5 0}-\mathbf{6}$ & 5,6 & 12,1 & 0,20 \\
\hline $\mathbf{4 - 7}$ & 3,3 & 5,5 & 0,10 \\
\hline $\mathbf{6}-\mathbf{7}$ & 5,0 & 7,0 & 0,18 \\
\hline $\mathbf{1 0}-\mathbf{7}$ & 7,0 & 8,5 & 0,26 \\
\hline $\mathbf{3 0}-\mathbf{7}$ & 6,6 & 11,0 & 0,27 \\
\hline $\mathbf{5 0}-\mathbf{7}$ & 6,8 & 13,2 & 0,28 \\
\hline & & &
\end{tabular}

En la Figura 7 se muestran las macrografías de los puntos obtenidos con 4, 10 y 50 ciclos, para la corriente de 6 KA.
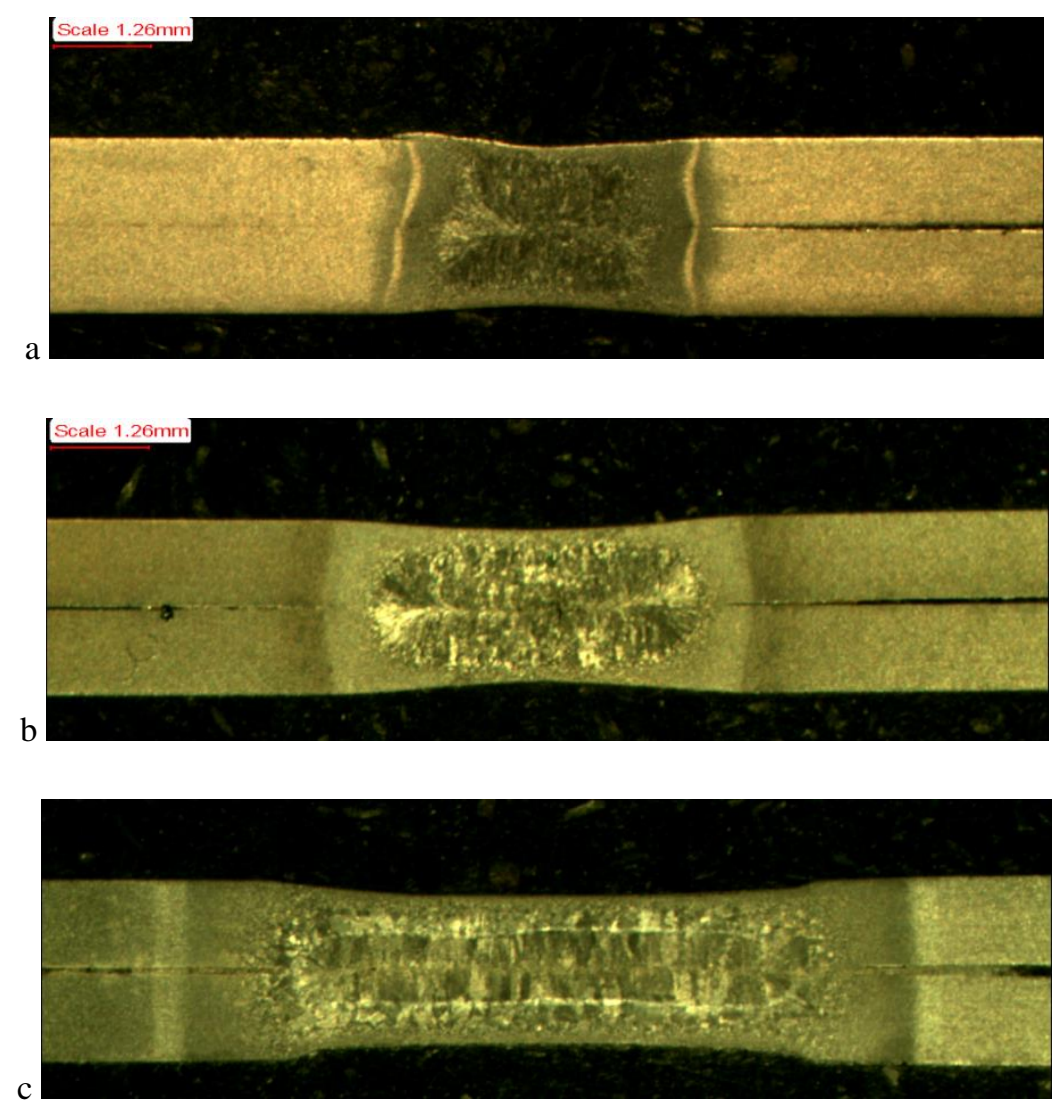

Figura 7: Macrografías de los puntos para la corriente media con: a-4, b-10 y c-50 ciclos

Como se puede apreciar el diámetro de la lenteja aumenta considerablemente con el incremento de los ciclos de soldadura y la altura de la misma disminuye, para una dada corriente. El nivel de expulsiones en la interface también aumenta, de la misma forma que la indentación.

En la Figura 8 se observa la evolución del ancho de la ZAC intercrítica y del diámetro de la lenteja de soladura (zona fundida) con el tiempo de soldadura y la corriente de soldadura analizada. 

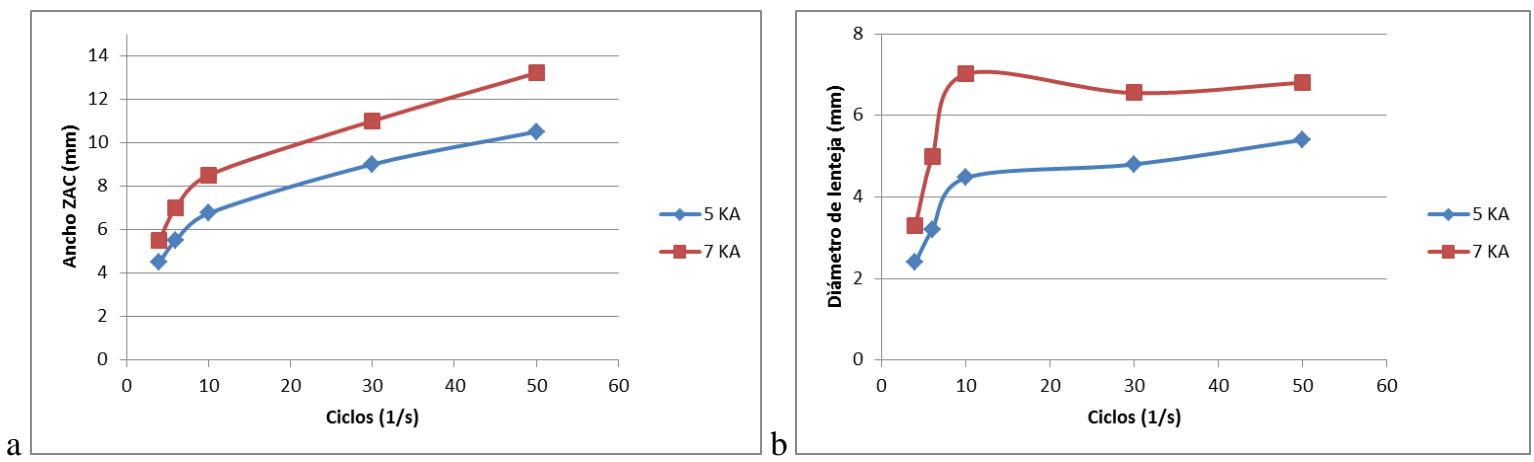

Figura 8: (a) Ancho de la ZAC intercrítica y (b) diámetro de la lenteja, en función de los ciclos de soldadura para los extremos de corriente analizados

Puede verse que el ancho de la ZAC aumenta con ambos parámetros. Asimismo, se ve que el diámetro de la lenteja también presenta un fuerte incremento hasta los 10 ciclos de soldadura para ambas corrientes, pero a mayores ciclos ya no se produce un mayor incremento dado que la cara activa de los electrodos es de 6 $\mathrm{mm}$.

\subsection{Caracterización microestructural de la junta soldada}

En la Figura 9 se muestran las micrografías de las distintas zonas de la junta soldada para el punto 10-6.

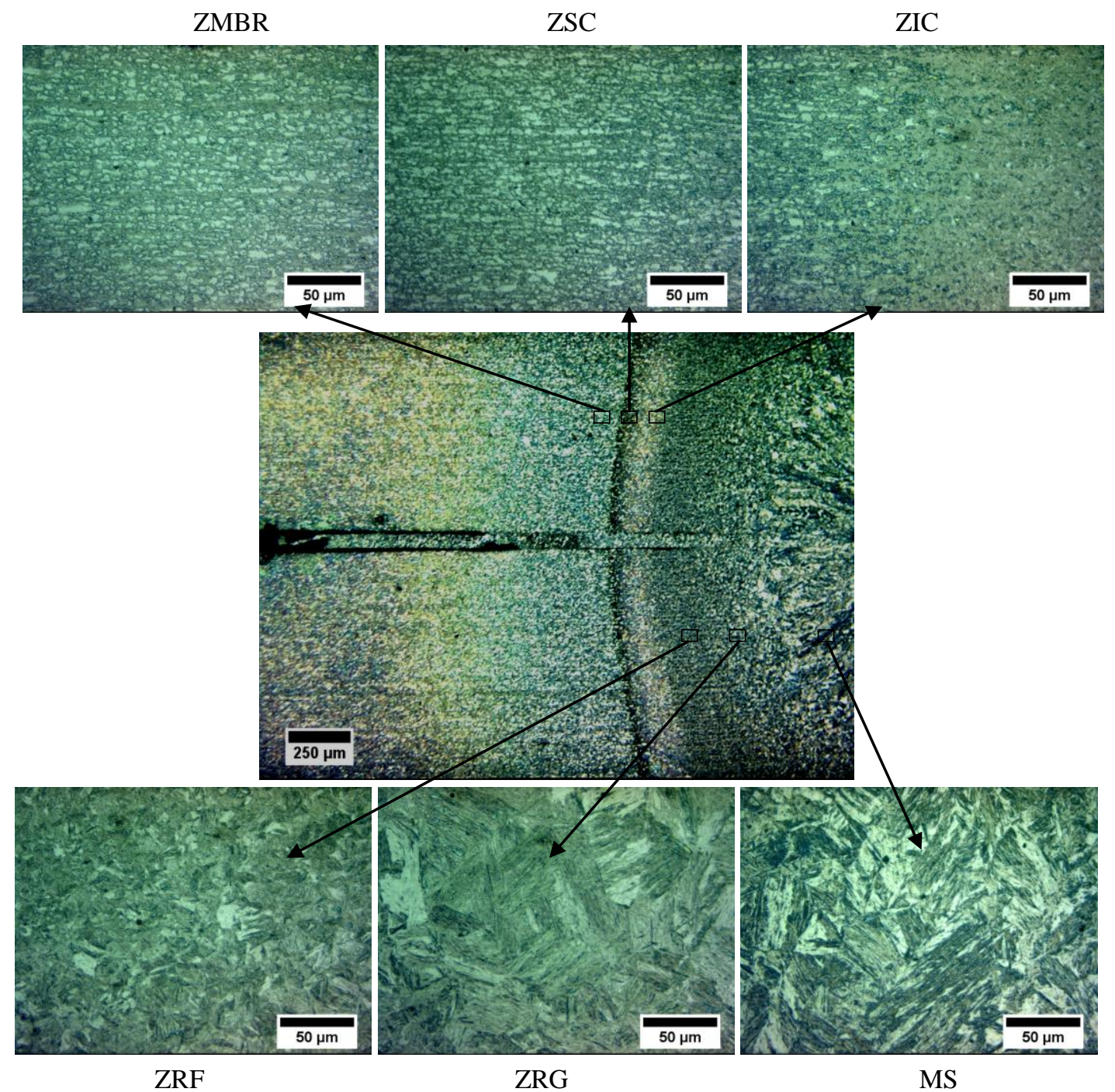

Figura 9: Micrografías de las distintas zonas del punto soldado 10-6 
Se observa en detalle la evolución en la ZAC desde la ZMBR (martensita revenida en matriz de ferrita), pasando luego por la ZSC (martensita revenida a mayor temperatura y de mínima dureza en matriz de ferrita), ZIC (ferrita y martensita fresca de tamaño muy fino y de máxima dureza), ZRF (martensita fresca de grano fino) y ZRG (martensita fresca de grano grueso), respectivamente. Finalmente el MS o lenteja de soldadura de estructura $100 \%$ martensítica.

En la Figura 10 se muestran con más detalles fotomicrografías de SEM de las tres zonas de las ZAC donde se produce el mayor cambio en microdureza.

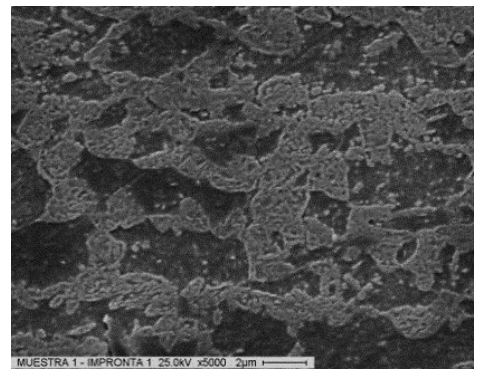

ZMBR $\left(\approx 280 \mathrm{HV}_{300}\right)$

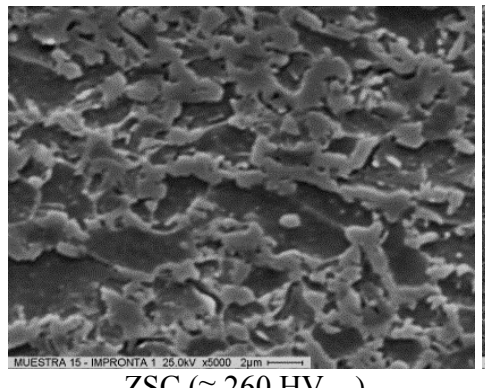

$\mathrm{ZSC}\left(\approx 260 \mathrm{HV}_{300}\right)$

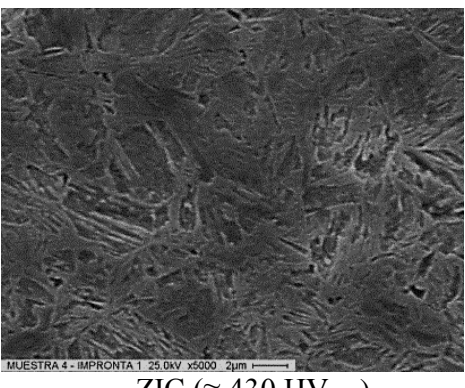

$\mathrm{ZIC}\left(\approx 430 \mathrm{HV}_{300}\right)$

Figura 10: Fotomicrografías SEM de la ZAC para el punto 10-6

\subsection{Perfiles de microdureza}

En la Figura 11 se muestran los perfiles de microdureza para 4, 10 y 50 ciclos y los dos niveles extremos de corriente 5 y $7 \mathrm{KA}$, respectivamente.

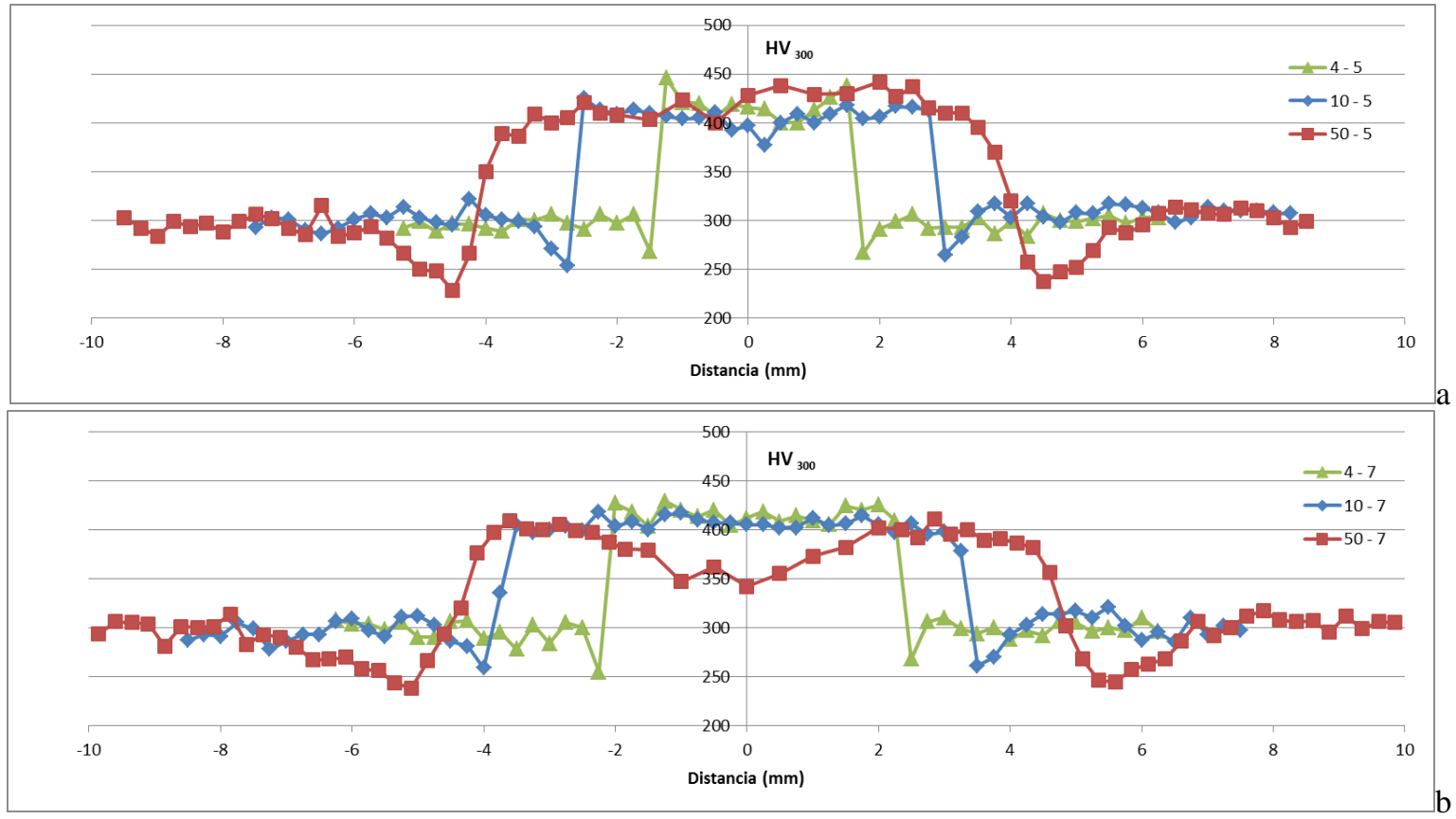

Figura 11: Perfiles de microdureza para $\operatorname{los} \operatorname{ciclos} 4,10$ y 50, con: a- 5 y b- 7 KA

Se observa que el ancho de la ZAC aumenta con los ciclos de soldadura para una dada corriente en mayor medida respecto a lo que lo hace con el aumento de corriente, para un dado ciclo. Tanto para corrientes bajas como altas el máxima ablandamiento aumenta con los ciclos de soldadura. Ese efecto de ablandamiento no se observa con el aumento de la corriente para un dado tiempo de soldadura. Para bajos ciclos las zona de mínima y máxima dureza se encuentran adyacentes (1 impronta - $25 \mu \mathrm{m}$ ), en cambio para el tiempos de soldadura mayores ese salto se da en una mayor distancia (hasta 6 improntas - $150 \mu \mathrm{m}$ ). Además la extensión de la ZMBR aumenta con el incremento de los ciclos. Este efecto estaría asociado a la variación del aporte térmico en función del tiempo y corriente de soldadura. Eso hace que para mayor aporte térmico, ma- 
yor el tiempo a elevada temperatura y menor la velocidad de enfriamiento [15]. En la muestra 50-7 se aprecia un ablandamiento en la zona central de la lenteja.

Los valores de dureza mínima oscilaron entre 230 y $260 \mathrm{HV}$, mientras que los de dureza máxima estuvieron entre 410 y $450 \mathrm{HV}$. Estos valores están asociados al revenido de la martensita a elevada temperatura, en el caso del mínimo, y a la formación de martensita fresca en el caso del máximo, debido a la elevada velocidad de enfriamiento impuesta por la alta tasa de extracción de calor de los electrodos. Un indicador de la degradación microestructural sufrida es el índice de dureza, definido como el cociente entre la dureza mínima y la del material base (309 HV). Los índices de dureza se encontraron entre 0,74 y 0,84.

\subsection{Ensayos mecánicos}

En la Tabla 5 se muestran los resultados de los ensayos mecánicos realizados.

Tabla 5: Resultados de los ensayos mecánicos

\begin{tabular}{l|l|l}
\hline MUESTRAS & $\begin{array}{l}\text { FUERZA MÁX. } \\
\text { PEEL (N) }\end{array}$ & $\begin{array}{l}\text { FUERZA MÁX. } \\
\text { CORTE (N) }\end{array}$ \\
\hline $\mathbf{4 - 5}$ & 410 & 6000 \\
\hline $\mathbf{6 - 5}$ & 1460 & 9850 \\
\hline $\mathbf{1 0}-\mathbf{5}$ & 1583 & 11350 \\
\hline $\mathbf{5 0}-\mathbf{5}$ & 1333 & 10650 \\
\hline $\mathbf{4}-\mathbf{7}$ & 1460 & 9450 \\
\hline $\mathbf{6}-\mathbf{7}$ & 1960 & 15250 \\
\hline $\mathbf{1 0}-\mathbf{7}$ & 2198 & 16700 \\
\hline $\mathbf{5 0}-\mathbf{7}$ & 1829 & 16100 \\
\hline
\end{tabular}

En la Figura 12 se muestran la evolución de la carga de rotura en los ensayos de peel y shear test en función de los ciclos de soldadura, para los extremos de corriente analizados (5 y $7 \mathrm{KA}$ ).
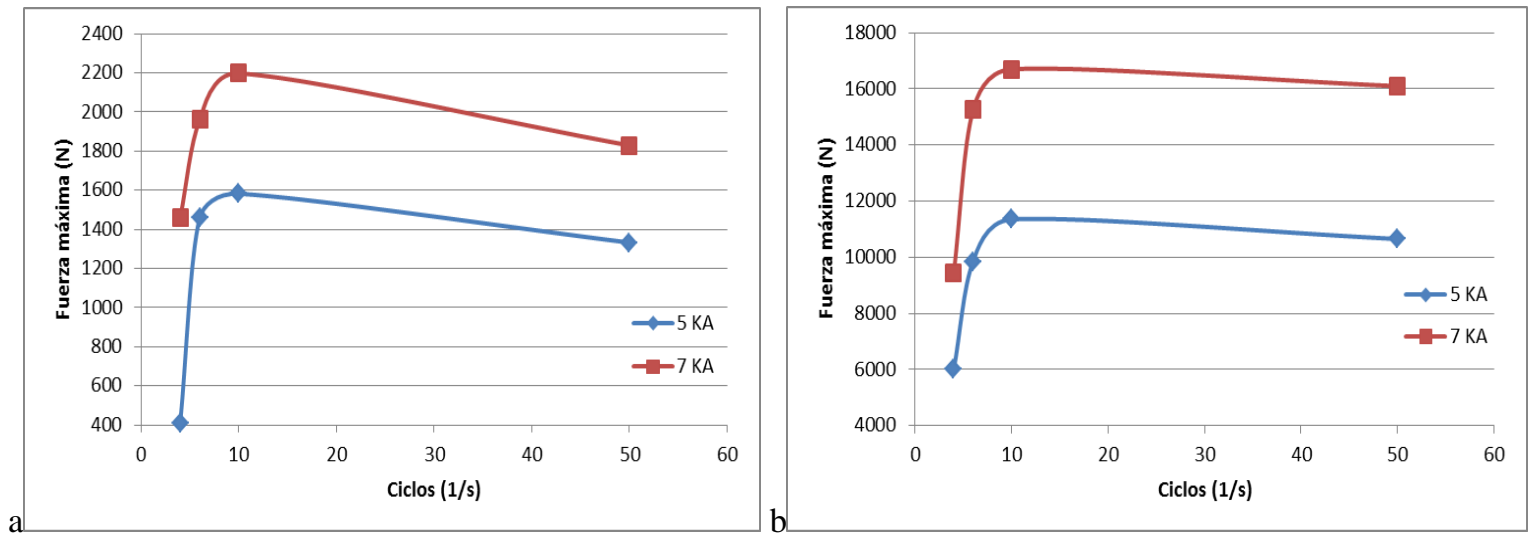

Figura 12: Carga de rotura de los ensayos de (a) peel y (b) shear test, en función de los ciclos de soldadura para los puntos con 5 y $7 \mathrm{KA}$

Se puede apreciar que la resistencia mecánica de los puntos analizados, tanto en arrancamiento como corte, alcanza un máximo en los 10 ciclos de soldadura. La pendiente inicial para bajos ciclos es muy fuerte indicando la gran influencia de este parámetro en las propiedades analizadas. Entre los 10 y 50 ciclos esa pendiente es mucho más suave manifestando que el óptimo se encontraría en ese rango. Respecto de la corriente también es notorio que a mayores niveles de la misma las propiedades mecánicas mejoran sustancialmente. Esto estaría asociado al aumento del tamaño de la lenteja con los ciclos y la corriente, lo que provee una mayor sección resistente. Asimismo, la presencia de defectos también afectaría la resistencia de la unión. Para mayores ciclos la indentación es mayor, así como el ablandamiento en la ZAC, lo que afectaría la carga de rotura de la unión. 


\subsection{Modos de fractura}

En las fotografías de la Figura 13 se muestran los distintos tipos de fractura del ensayo de peel test para los puntos analizados.

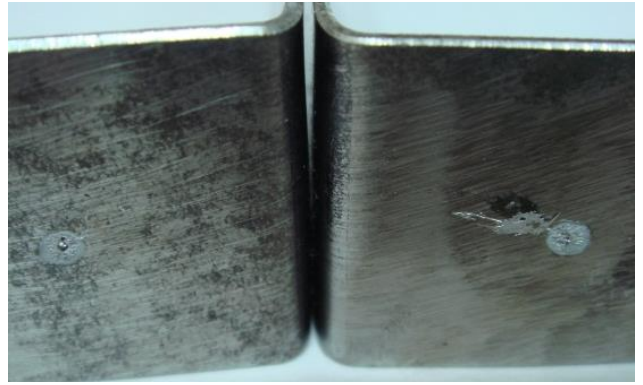

4-5 (desprendimiento)

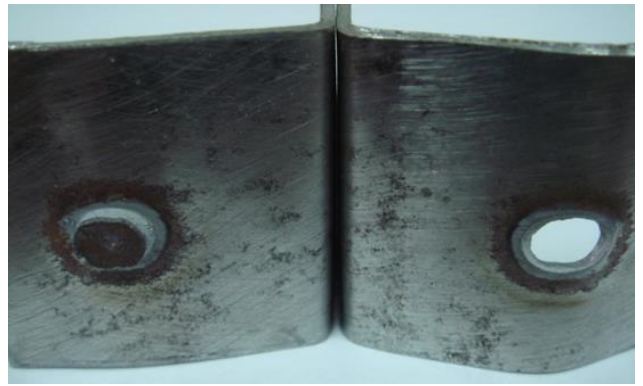

10-5 (arranque total)

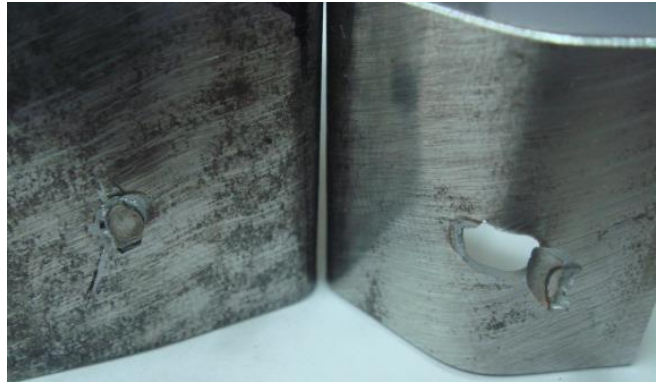

4-6 (arranque parcial)

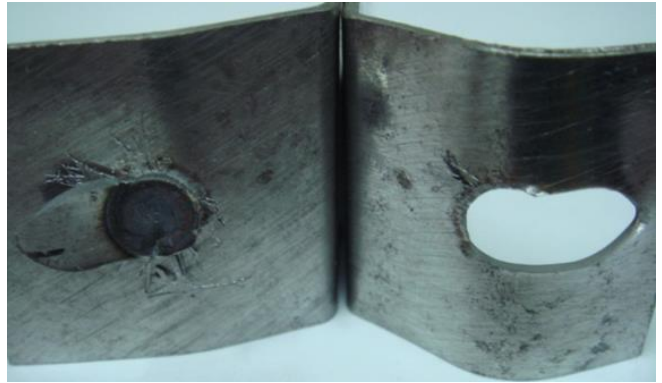

50-7 (arranque total con arrastre de MB)

Figura 13: Fotografías con los diferentes modos de fractura del ensayo de peel analizados.

En las macrografías de la Figura 14 se muestran los cortes transversales a la fractura de los puntos

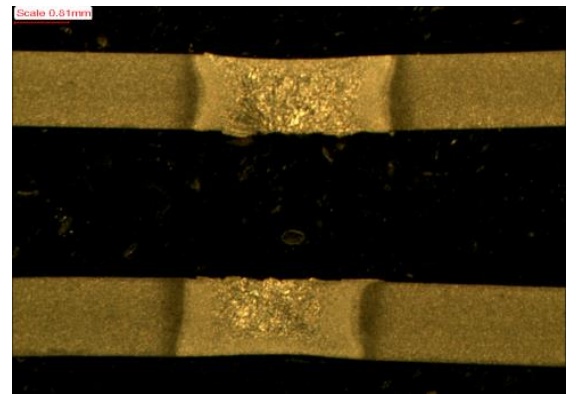

4-5 (desprendimiento)

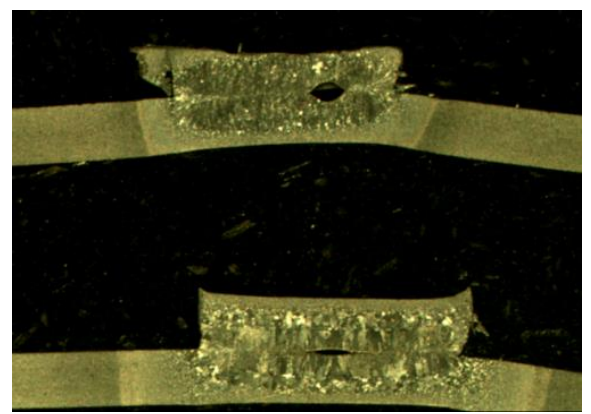

$10-5$ y $50-5$

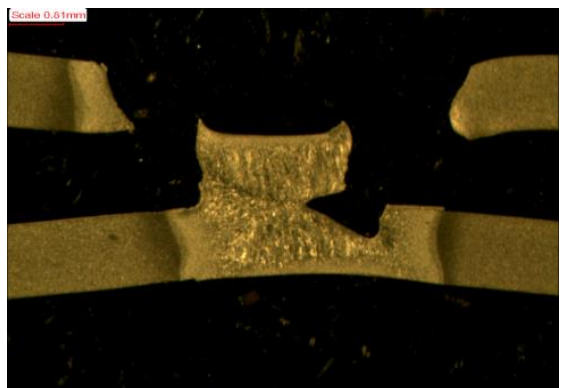

4-6 (arranque parcial)

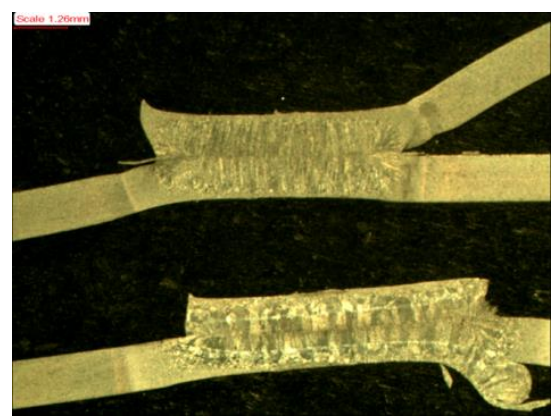

10-7 y $50-7$

Figura 14: Macrografías de los puntos ensayados por peel test 
Se observa como evoluciona favorablemente el modo de fractura, desde el desprendimiento interfacial (baja carga de rotura), pasando por un arranque parcial hasta el arranque total (alta carga de rotura), con el aumento de los ciclos y corriente de soldadura. Puede verse que la fractura evoluciona por la ZAC desde la línea de fusión en general o desde la zona blanda, algo más alejada de dicha línea de fusión.

En las fotografías de la Figura 15 se muestran los distintos tipos de fractura del ensayo de shear test para los puntos analizados.

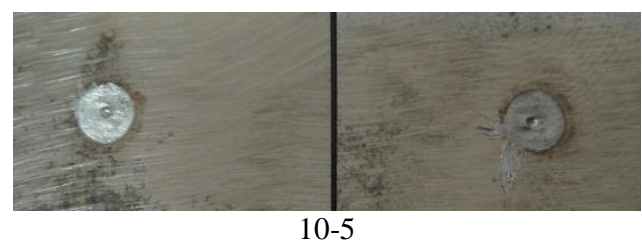

$10-5$

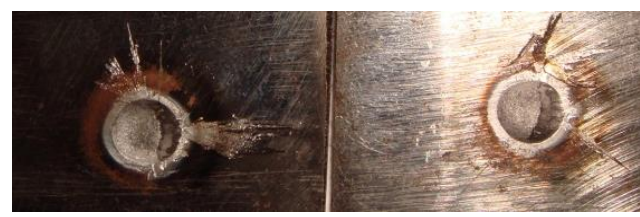

$10-7$
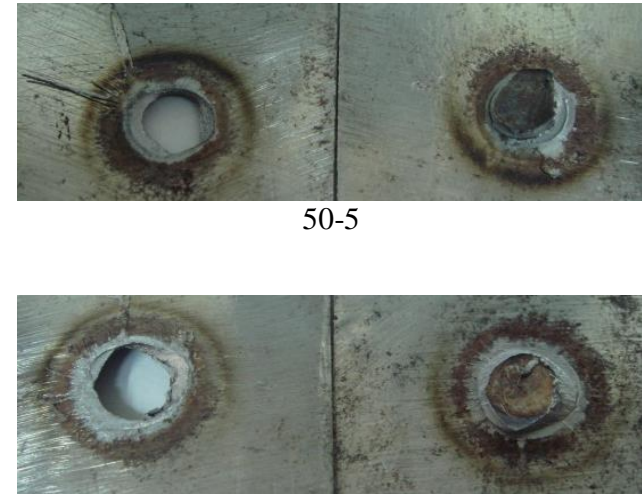

$50-7$

Figura 15: Fotografías con los diferentes modos de fractura del ensayo de shear test

En la Figura 16 se muestran las macrografías de los ensayos de shear test para los puntos analizados.

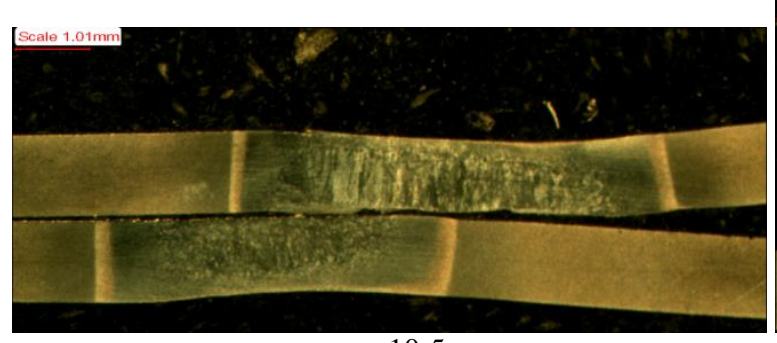

$10-5$

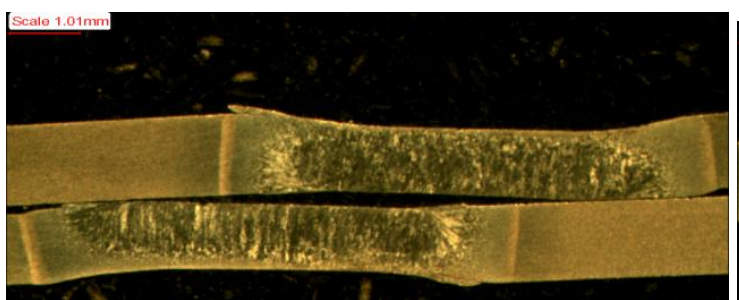

$10-7$

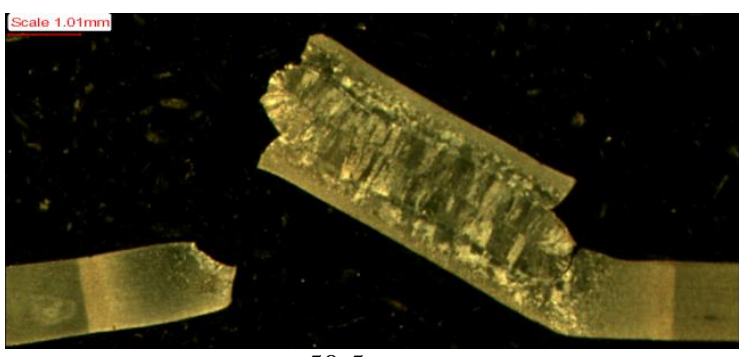

$50-5$

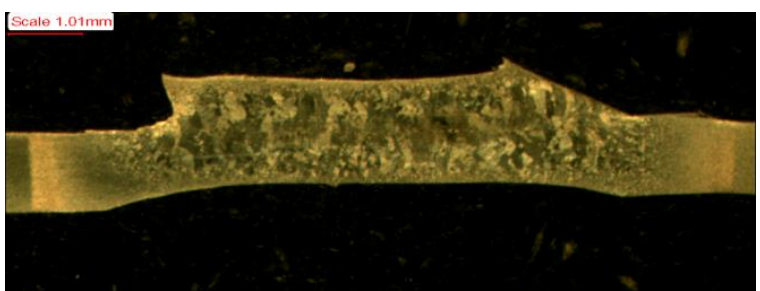

$50-7$

Figura 16: Macrografías de los puntos ensayados por shear test

En este caso se aprecian dos tipos de fractura debido al ensayo de corte. Por un lado interfacial para los 10 ciclos y menores, y por el otro de arranque total para los ciclos más altos, tanto para las bajas como altas corrientes analizadas. Es probable que para esta condición de ensayo el cambio en el modo de fractura, y por lo tanto el óptimo en cuanto al valor de la carga de rotura se encuentre para una número de ciclos mayor que 10 y menor que 50 .

Como se mencionó anteriormente, el aumento del número de ciclos de 10 a 50 produce una disminución de la carga de rotura en ambos ensayos. Esto se asocia a que el diámetro de la lenteja no aumenta debido a que se alcanzó el límite para la cara activa del electrodo, mientras que la indentación aumenta levemente así como la degradación metalúrgica (ablandamiento). Esto hace que la sección resistente del ligamento circunferencial por donde evoluciona la fractura disminuya (Figuras 14 y 16), así como su resistencia, produciendo una disminución de la carga necesaria para producir su rotura. 


\section{DISCUSIÓN}

Con los resultados observados y correlacionando con la bibliografía disponible para este proceso de soldadura y este tipo de aceros se puede aportar la siguiente discusión:

En primer lugar los bajos ciclos de soldadura planteados (4 y 6) no satisfacen los requerimientos mínimos adoptados por la industria automotriz en cuanto al diámetro de la lenteja de soldadura establecido en $4 \sqrt{t}=4,38 \mathrm{~mm}$, donde $\mathrm{t}$ es el espesor del metal base. A partir de los 10 ciclos de soldadura se puede decir que se cumple este requisito para todas las corrientes analizadas. Mayores corrientes no proporcionan un mayor grado de aceptación debido a que se alcanza el límite impuesto por el diámetro de la cara activa del electrodo.

En cuanto a las propiedades mecánicas ellas están íntimamente relacionadas con ese requisito de diámetro mínimo de la lenteja, y se manifiesta notoriamente con los distintos modos de fractura observados. Pero además el aumento de los ciclos y la corriente produce un incremento en el nivel de expulsiones entre las chapas, lo que conlleva a que los inicios de las fracturas se produzcan en diferentes zonas y luego se propaguen de manera distinta según la microestructura resultante, producto de los parámetros de ciclos y corrientes establecidos para cada punto. Esto ha sido objeto de estudio por varios investigadores y se continúa actualmente en la predicción del comportamiento a fatiga y estructural de estos aceros soldados por resistencia [16]. Sin embargo, la rápida evolución de los mismos hace necesaria la verificación experimental, dada la notoria influencia de las variables puestas en juego, y sobre todo conociendo el gran efecto que produce el ciclo térmico de la soldadura en este tipo de AHSS, como puede apreciarse en la evolución microestructural y en los respectivos perfiles de microdureza.

\section{CONCLUSIONES}

Con el esquema de trabajo planteado se pudo establecer un juego de parámetros óptimos para la soldadura de aceros DP de $1000 \mathrm{MPa}$ de resistencia de $1,2 \mathrm{~mm}$ de espesor. Se encontró que a partir de los 10 ciclos de soldadura y con niveles de corriente de 7 KA los puntos cumplen con los requerimientos dimensionales y de propiedades mecánicas adoptados por la industria automotriz. Mayores incrementos de estos parámetros aseguran el cumplimiento de los requisitos pero se contrarrestan en cuanto a los costos asociados al proceso de soldadura en cuestión, como puede ser la vida útil de los electrodos o el aspecto superficial de los puntos, entre otros aspectos económicos y de calidad respectivamente.

De todas formas se deja planteada, para trabajos futuros, la necesidad de ajustar más aún la ventana de parámetros de soldadura propuesta para encontrar el punto óptimo.

\section{AGRADECIMIENTOS}

Los autores agradecen a SSAB y su distribuidora Brugsa S.A. en Argentina por la donación de las chapas de acero DP1000; y a los laboratorios de INTI Mecánica y de la Escuela de Tecnología de la UNNOBA por la realización de los ensayos de peel y shear test. A INTI Mecánica por las imágenes SEM. Al centro DEYTEMA y el departamento de Metalurgia de la FRSN por permitir el uso de los laboratorios. También agradecen el apoyo financiero de la Universidad Tecnológica Nacional y en especial a la Fundación Hermanos Rocca del Grupo Techint por el apoyo económico para la adquisición del equipamiento de soldadura.

\section{BIBLIOGRAFÍA}

[1] JOOST, W.J., "Reducing vehicle weight and improving U.S. energy efficiency using integrated computational materials engineering”, JOM, v. 64, n. 9, pp. 1032-1038, Sep. 2012.

[2] SHOME, M., TUMULURU, M., "Introduction to welding and joining of advanced high-strength steels (AHSS)", In Shome, M. and Tumuluru, M. (eds), Welding and Joining of Advanced High Strength Steels (AHSS), 1 ed., chapter 1, Sawston, Cambridge, UK, Woodhead Publishing Series in Welding and Other Joining Technologies, n. 85, 2015.

[3] HILDITCH, T.B., SOUZA, T., HODGSON, P.D., "Properties and automotive applications of advanced high-strength steels (AHSS)", In Shome, M. and Tumuluru, M. (eds), Welding and Joining of Advanced High Strength Steels (AHSS), 1 ed., chapter 2, Sawston, Cambridge, UK, Woodhead Publishing Series in Welding and Other Joining Technologies, n. 85, 2015.

[4] TUMULURU, M., "Resistance spot welding techniques for advanced high-strength steels (AHSS)", In Shome, M. and Tumuluru, M. (eds), Welding and Joining of Advanced High Strength Steels (AHSS), 1 ed., chapter 3, Sawston, Cambridge, UK, Woodhead Publishing Series in Welding and Other Joining Technologies, n. 85, 2015. 
[5] BALTAZAR HERNANDEZ,V.H., PANDA, S.K., KUNTZ, M.L., et al., "Nanoindentation and microstructure analysis of resistance spot welded dual phase steel”, Materials Letters, v. 64, n. 2, pp. 207-210, Ene. 2010.

[6] SVOBODA, H., LORUSSO, H.N., BURGUEÑO, A., "Soldadura de aceros Dual Phase en chapa fina: GMAW, PAW y RSW”, Soldagem \& Inspeção, v. 16, n. 2, pp.165-176, Abr/Jun. 2011.

[7] MA, C., CHEN, D.L., BHOLE, S.D., BOUDREAU, G., et al., "Microstructure and fracture characteristics of spot welded DP600 steel”, Materials Science and Engineering: A, v. 485, n. 1-2, pp. 334-346, Jun. 2008.

[8] MARYA, M., GAYDEN, X., "Development of requirement for Resistance Spot Welding Dual-Phase (DP600) Steels Part 1-The cause of interfacial fracture", Welding Journal, v. 84, n. 11, pp. 172s-185s, Nov. 2005.

[9] RAMAZANI, A., MUKHERJEE, K., ABDURAKHMANOV, A., et al., "Characterization of Microstructure and Mechanical Properties of Resistance Spot Welded DP600 Steel”, Metals, v. 5, pp. 1704-1716, Sep. 2015.

[10] HUIN, T., DANCETTE, S., FABRÈGUE, D., DUPUY, T., "Investigation of the Failure of Advanced High Strength Steels Heterogeneous Spot Welds”, Metals, v. 6, n. 111, pp. 1-19, May. 2016.

[11] SUN, X., STEPHENS, E.V., KHALEEL, M.A., "Effects of Fusion Zone Size and Failure Mode on Peak Load and Energy Absorption of Advanced High-Strength Steel Spot Welds", Welding Journal, v. 86, n. 01, pp. 18s-25s, Ene. 2007.

[12] ISO 18278-2:2004, "Resistance welding - Weldability - Part 2: Evaluation procedures for weldability in spot welding", 2004.

[13] ISO 14270:2000, "Resistance welding - Destructive testing of welds - Specimen dimensions and procedure for mechanized peel testing resistance spot, seam and embossed projection welds", 2000.

[14] ISO 14273:2000, "Resistance welding - Destructive testing of welds - Specimen dimensions and procedure for tensile shear testing resistance spot and embossed projection welds", 2000.

[15] CONNOR, L.P., Welding Handbook, Vol. 1: Welding science and technology, 9 ed., Miami, FL, USA, American Welding Society, 2001.

[16] VANIMISETTI, S.K., SIGLER, D.R., "Improving Fatigue Performance of Spot Welds in Advanced High-Strength Steels", Welding Journal, v. 93, n. 05, pp. 153s-161s, May. 2014. 